\title{
Asymptotics for models of non-stationary diffusion in domains with a surface distribution of obstacles
}

\author{
Delfina Gómez ${ }^{(1)}$, Miguel Lobo ${ }^{(1)}$, Maria-Eugenia Pérez-Martínez ${ }^{(2), \star}$ \\ (1) Departamento de Matemáticas, Estadística y Computación, \\ Universidad de Cantabria, Santander, Spain \\ (2) Departamento de Matemática Aplicada y Ciencias de la Computación, \\ Universidad de Cantabria, Santander, Spain \\ * Corresponding author: meperez@unican.es
}

\begin{abstract}
We consider a time-dependent model for the diffusion of a substance through an incompressible fluid in a perforated domain $\Omega_{\varepsilon}, \Omega_{\varepsilon} \subset \Omega \subset \mathbb{R}^{n}$ with $n=3$, 4. The fluid flows in a domain containing a periodical set of "obstacles" $\left(\Omega \backslash \Omega_{\varepsilon}\right)$ placed along an inner $(n-1)$-dimensional manifold $\Sigma \subset \Omega$. The size of the obstacles is much smaller than the size of the characteristic period $\varepsilon$. An advection term appears in the partial differential equation linking the fluid velocity with the concentration, while we assume a nonlinear adsorption law on the boundary of the obstacles. This law involves a monotone nonlinear function $\sigma$ of the concentration and a large adsorption parameter. The "critical adsorption parameter" depends on the size of the obstacles and, for different sizes, we derive the time-dependent homogenized models. These models contain a "strange term" in the transmission conditions on $\Sigma$, which is a nonlinear function and inherits the properties of $\sigma$. The case in which the fluid velocity and the concentration do not interact is also considered for $n \geq 3$.
\end{abstract}

Keywords: Boundary homogenization; evolution problems; nonlinear problems; asymptotic expansions; critical parameters.

\section{Introduction}

In this paper, we describe the asymptotic behavior, as $\varepsilon \rightarrow 0$, of the solution of a coupled fluid-diffusion problem in a perforated domain. The model under consideration (cf. (5)(6)) addresses the diffusion of a substance through an incompressible fluid in a domain $\Omega_{\varepsilon}$ of $\mathbb{R}^{n}, n=3,4 . \Omega_{\varepsilon}$ is a periodically perforated domain along an inner $(n-1)$-dimensional manifold $\Sigma \subset \Omega$ (cf. Figure 1): $\Omega$ is a bounded domain with a smooth boundary and $\Omega_{\varepsilon}$ denotes $\Omega$ minus the set of obstacles. Mathematically, the obstacles are represented by holes or perforations, but from a physical viewpoint they are solid inclusions where the fluid cannot flow. The periodicity of the structure is described by a small parameter $\varepsilon$. The obstacles are assumed to be domains of $\mathbb{R}^{n}$, homothetics of a fixed domain, $G_{0}$; they have a smooth boundary and a diameter $O(r(\varepsilon))$ with $r(\varepsilon) \ll \varepsilon$. For the diffusion problem, a nonlinear law is prescribed on their boundary with a large adsorption parameter $\beta(\varepsilon)$. 
Further specifying, the velocity of the fluid, which we denote by $\vec{v}_{\varepsilon}$, is assumed to be null on the boundary of the perforated domain $\partial \Omega_{\varepsilon}$ and independent of the concentration of solute $u_{\varepsilon}$, cf. (5). $\vec{v}_{\varepsilon}$ is also assumed to be time-independent. $u_{\varepsilon} \equiv u_{\varepsilon}(x, t)$ is the solution of an initial-boundary value problem for a parabolic equation in $\Omega_{\varepsilon}$ satisfying a Dirichlet condition on $\partial \Omega$. On the boundary of the obstacles, $u_{\varepsilon}$ satisfies a nonlinear Robin condition (cf. (6)), the nonlinear term being $\beta(\varepsilon) \sigma\left(x, u_{\varepsilon}\right)$ where $\sigma$ is a monotone function of the concentration (cf. (2)-(4) and item C4 in Section 4). An advection term in the partial differential equation relates both velocity and concentration, namely, $\operatorname{div}\left(u_{\varepsilon} \vec{v}_{\varepsilon}\right)$. We assume that $\beta(\varepsilon)$ and $r(\varepsilon)$ are functions of $\varepsilon$, which are related by (1): that is, by the fact that the adsorption parameter multiplied by the total area of the obstacles is of order $O(1)$. For different relations between $\varepsilon, \beta(\varepsilon)$ and $r(\varepsilon)$, we obtain the homogenized models (cf. (9) (10)). We refer to [10] for a precise description of the physical model under consideration in the stationary case. See also [13], and references therein, in connection with these kinds of models in perforated domains over the whole volume.

The case where $r(\varepsilon)=O\left(\varepsilon^{\frac{n-1}{n-2}}\right)$ gives the classical critical size for obstacles while the relation $\beta(\varepsilon)=O\left(\varepsilon^{n-1} r(\varepsilon)^{1-n}\right)$ provides a so-called critical relation for the adsorption parameter, the critical adsorption parameter for a given size of obstacles. For these relations, in general, there appears asymptotically a strange term in the transmission conditions on $\Sigma$ for the derivatives of the solutions $\vec{v}_{\varepsilon}$ and $u_{\varepsilon}$, respectively. The homogenization of the Stokes problem only depends on $r(\varepsilon)$ and, as is well-known in the literature (cf., e.g., $[1,3,10])$, the strange term only appears for the critical size of the obstacles (that is $\left.r(\varepsilon)=O\left(\varepsilon^{\frac{n-1}{n-2}}\right)\right)$ and it involves the so-called Stokes capacity matrix (cf. (14) and (15)).

For the concentration, we use the technique of matched asymptotic expansions to derive the homogenized problem (cf. Section 3.1). The strange term obtained in the homogenization process is different depending on the relation between the two parameters $\beta(\varepsilon)$ and $r(\varepsilon)$. The most critical situation occurs when simultaneously we have the classical critical size of the obstacles and the critical adsorption parameter. In this case, the strange term is defined through the "average" expression (12), which is referred to as (cf. [10]) extended capacity, and involves the nonlinear function $\sigma$ and the parameter $\beta_{0}$ (cf. (10)) relating the ratio of the obstacles and the adsorption: see the homogenized problem (11) and the bi-parametric family of local problems (13). In the case of very big obstacles, the strange term contains the area of the obstacles in the averaged constants accompanying $\sigma$ : cf. the homogenized problem (21). In the case of very small obstacles, the homogenized problem ignores both obstacles and adsorption: cf. (19). For spherical obstacles, computations on the local problem simplify (cf. (40)) and, in the most critical situation, the strange term can be defined through a nonlinear function implicitly defined from a functional equation (cf. (38) and (39)). We refer to [7, 11, 12, 15, 19, 20] for critical sizes and strange terms in linear and nonlinear problems, and for further references. See $[6,8]$ for some critical relations of parameters on the boundary conditions of fluid models in porous media. For recent works on non-stationary diffusion models in porous media cf., e.g., $[2,5,13]$ and references therein.

Let us mention that stationary problems associated with our model have been addressed in $[4,10]$, where an extensive bibliography on the model and previous works is provided. In this paper, we give results that extend and complement those in [4] and [10] to arbitrary shapes of the obstacles and to the dimension $n>3$, respectively. Fur- 
ther specifying, Ref. [4] addresses the convergence in the most critical situation of the time-independent scalar problem for a domain perforated by balls. The model here considered represents an improvement with respect to that of [4] since the adsorption process is time-dependent and we deal with arbitrary shapes of the obstacles. Ref. [10] addresses the stationary model for a general geometry of the obstacles, in dimension 3 , and for all the relations between the parameters, when a non-null velocity of the fluid is prescribed on the outer boundary $\partial \Omega$. This non-null velocity makes it difficult to obtain the convergence of the solution of the Stokes problem: see [9, 10, 20, 21] and references therein, in this respect. The computations in [10] are based on asymptotic expansions, both for the homogenization of the Stokes problem and for the scalar nonlinear stationary problem. Here, we use the technique in [10] to derive the scalar evolution homogenized problems for the dimension of the space $n \geq 3$. We emphasize that the results in this paper are new in the literature; also, they are in good agreement with those obtained in [10] when $n=3$ and $u_{\varepsilon}$ does not depend on $t$. Also, we emphasize that the proof of the convergence of $u_{\varepsilon}$, as $\varepsilon \rightarrow 0$, is currently the subject of research: see items C3 and C5 in Section 4 in this connection.

Finally, the paper is structured as follows: In Section 2, we introduce the description of the geometry of the problem and the homogenization problems (5) and (6) for the velocity of the fluid $\vec{v}_{\varepsilon}$ and for the concentration $u_{\varepsilon}$. In Section 3, depending on the different relations between parameters, we state the corresponding homogenized problems (14), (20) and (22), and (11), (19) and (21), respectively. Section 3.1 contains a sketch of proofs. Due to the advection term, the results hold for $n=3,4$, (cf. (34)). However, the technique in Section 3.1 also applies to $n>4$, when further restrictions on $\vec{v}_{\varepsilon}$ are performed or the advection term does not appear, or for associated stationary problems. In Section 4, we deal with these problems (cf. items C1 and C2) and we provide some comments on the geometrical configuration, the nonlinear adsorption law, the convergence and open problems.

\section{Setting of the problem}

Let $\Omega$ be a bounded domain in $\mathbb{R}^{n}, n \geq 3$, with a smooth boundary $\partial \Omega$. Assume that $\Sigma=\Omega \cap\left\{x_{1}=0\right\} \neq \emptyset$ divides $\Omega$ in two parts $\Omega^{+}=\Omega \cap\left\{x_{1}>0\right\}$ and $\Omega^{-}=\Omega \cap\left\{x_{1}<0\right\}$ (cf. Figure 1). We denote by $G_{0}$ a domain of $\mathbb{R}^{n}$, of positive volume $\left|G_{0}\right|>0$, with a smooth boundary and we assume that it contains the origin of the coordinates.

Let $\varepsilon$ be a small parameter $\varepsilon \ll 1$, and $r(\varepsilon)$ be an order function such that $r(\varepsilon) \ll \varepsilon$. For a domain $B$, and for $a>0$, we denote by $a B=\left\{x \mid a^{-1} x \in B\right\}$. For $\mathbf{j} \in \mathbb{Z}^{\prime}$, we set $G_{\varepsilon}^{\mathbf{j}} \equiv r(\varepsilon) G_{0}+\varepsilon \mathbf{j}$, where $\mathbb{Z}^{\prime}$ is the set of points of the form $\mathbf{j}=\left(0, z_{2}, \ldots, z_{n}\right)$ with integer components $z_{i}, i=2, \ldots, n$. In what follows, if no confusion arises, we identify $\mathbf{j} \in \mathbb{Z}^{\prime}$ with $j \in \mathbb{Z}^{\prime}$ and denote $G_{\varepsilon}^{\mathrm{j}}$ by $G_{\varepsilon}^{j}$. Let $\widetilde{x}_{\varepsilon}^{\mathrm{j}}$ denote the center of $G_{\varepsilon}^{j}$. We define $G_{\varepsilon}$ as

$$
G_{\varepsilon}=\bigcup_{j \in \Upsilon_{\varepsilon}} G_{\varepsilon}^{j}
$$

where $\Upsilon_{\varepsilon}=\left\{\mathbf{j} \in \mathbb{Z}^{\prime}: \bar{G}_{\varepsilon}^{\mathbf{j}} \subset \Omega, \rho\left(\partial \Omega, \bar{G}_{\varepsilon}^{\mathbf{j}}\right) \geq 2 \varepsilon\right\}$ and $\rho$ denotes the usual distance between sets of $\mathbb{R}^{n}$. Let us denote by $N(\varepsilon)$ the number of $G_{\varepsilon}^{j}$ with index $j \in \Upsilon_{\varepsilon}: N(\varepsilon) \equiv\left|\Upsilon_{\varepsilon}\right|$ is 
of order $\varepsilon^{1-n}$ (cf. Figure 1). Finally, for the domains, we set

$$
\Omega_{\varepsilon}=\Omega \backslash \bar{G}_{\varepsilon}, S_{\varepsilon}=\partial G_{\varepsilon}, \partial \Omega_{\varepsilon}=\partial \Omega \cup S_{\varepsilon} .
$$
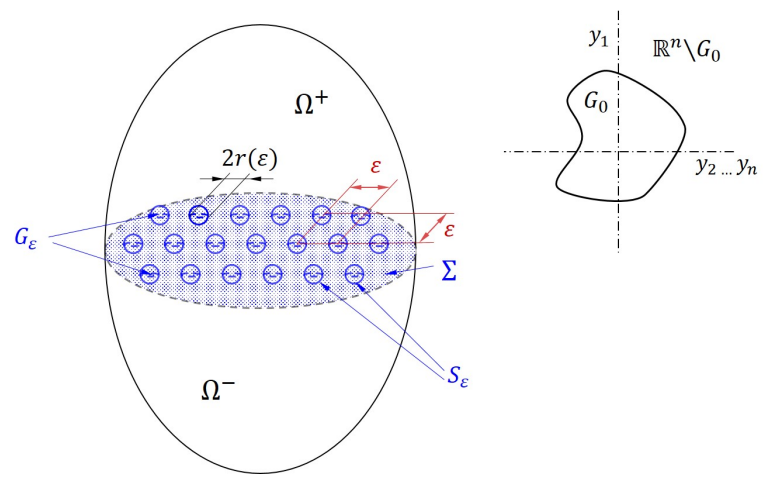

Figure 1: The geometrical configuration of $\Omega_{\varepsilon}$.

Let us introduce the functions $\beta(\varepsilon)$ and $\sigma(x, u)$ which appear in the boundary conditions of the problem.

Let $\beta(\varepsilon)$ be a positive function of the parameter $\varepsilon$ satisfying

$$
\lim _{\varepsilon \rightarrow 0} \beta(\varepsilon) \varepsilon^{1-n} r(\varepsilon)^{n-1}=\beta^{*}>0 .
$$

Let us consider $\sigma(x, u)$ a continuous function in $\bar{\Omega} \times \mathbb{R}$ satisfying

$$
\sigma(x, 0)=0, \quad(\sigma(x, u)-\sigma(x, v))(u-v) \geq 0, \quad \forall x \in \bar{\Omega}, \quad u, v \in \mathbb{R},
$$

and that there are constants $\tilde{k}_{1}>0, \delta$ and $\gamma$ such that, $\forall u \in \mathbb{R}$,

$$
|\sigma(x, u)| \leq \tilde{k}_{1}\left(|u|+|u|^{\delta+1}\right), \quad \text { with } \quad \delta \in[0,2 /(n-2)]
$$

or

$$
|\sigma(x, u)| \leq \tilde{k}_{1}|u|^{\gamma}, \quad \text { with } \quad 0<\gamma<1 .
$$

See also item $\mathrm{C} 4$ in Section 4 for weaker restrictions on $\sigma$.

For $\vec{F} \in\left(L^{2}(\Omega)\right)^{n}$ and $f \in L^{2}\left(0, T ; L^{2}(\Omega)\right)$, with any fixed $T>0$, we consider the solutions $\left(\vec{v}_{\varepsilon}, p_{\varepsilon}\right)$ and $u_{\varepsilon}$ of the following problems: the Stokes fluid flow problem

$$
\begin{cases}-\mu \Delta \vec{v}_{\varepsilon}+\nabla p_{\varepsilon}=\vec{F} & \text { in } \Omega_{\varepsilon}, \\ \operatorname{div}\left(\vec{v}_{\varepsilon}\right)=0 & \text { in } \Omega_{\varepsilon}, \\ \vec{v}_{\varepsilon}=0 & \text { on } \partial \Omega_{\varepsilon},\end{cases}
$$

with the viscosity $\mu>0$, and the semilinear evolution problem

$$
\begin{cases}\frac{\partial u_{\varepsilon}}{\partial t}-\Delta u_{\varepsilon}+\operatorname{div}\left(u_{\varepsilon} \vec{v}_{\varepsilon}\right)=f & \text { in } \Omega_{\varepsilon} \times(0, T) \\ \frac{\partial u_{\varepsilon}}{\partial \nu}+\beta(\varepsilon) \sigma\left(x, u_{\varepsilon}\right)=0 & \text { for }(x, t) \in S_{\varepsilon} \times[0, T] \\ u_{\varepsilon}(x, t)=0 & \text { for }(x, t) \in \partial \Omega \times[0, T] \\ u_{\varepsilon}(x, 0)=0 & \text { for } x \in \Omega_{\varepsilon}\end{cases}
$$


with $n=3,4$, and $\nu$ the unit outward normal vector to $\partial \Omega_{\varepsilon}$ on $S_{\varepsilon}$. See Section 4 for $n>4$.

For the given force $\vec{F}, \vec{v}_{\varepsilon}$ represents the velocity of the flow and $p_{\varepsilon}$ the pressure when a Dirichlet boundary condition is prescribed on the whole boundary $\partial \Omega_{\varepsilon}$. As is well known, the existence and uniqueness of solution $\left(\vec{v}_{\varepsilon}, p_{\varepsilon}\right) \in\left(H_{0}^{1}\left(\Omega_{\varepsilon}\right)\right)^{n} \times L^{2}\left(\Omega_{\varepsilon}\right) / \mathbb{R}$ of problem (5) is a consequence of the general theory for the Stokes problem (cf., e.g., $[16,22])$.

Similarly $u_{\varepsilon}$ represents, e.g., the concentration of a polluting substance in the fluid, with the adsorption law on the boundary of the inclusions given by the nonlinear Robin boundary condition on $S_{\varepsilon}$ in problem (6) and the adsorption parameter $\beta(\varepsilon)$ is assumed to satisfy the relation (1); that is, it is of the same order of magnitude as the inverse of the total area of the obstacles $G_{\varepsilon}$.

Let us recall the definition of the weak solution of the evolution problem: for any fixed $T>0$, a function $u_{\varepsilon} \in L^{2}\left(0, T ; H^{1}\left(\Omega_{\varepsilon}, \partial \Omega\right)\right), \partial_{t} u_{\varepsilon} \in L^{2}\left(0, T ;\left(H^{1}\left(\Omega_{\varepsilon}, \partial \Omega\right)\right)^{\prime}\right)$ is a weak solution of problem (6) if the following equality holds

$$
\begin{array}{r}
\left\langle\partial_{t} u_{\varepsilon}, v\right\rangle+\int_{\Omega_{\varepsilon}} \nabla u_{\varepsilon} \cdot \nabla v d x+\beta(\varepsilon) \int_{S_{\varepsilon}} \sigma\left(x, u_{\varepsilon}\right) v d s_{x}-\int_{\Omega_{\varepsilon}} u_{\varepsilon} \vec{v}_{\varepsilon} \cdot \nabla v d x=\int_{\Omega_{\varepsilon}} f v d x, \\
\forall v \in H^{1}\left(\Omega_{\varepsilon}, \partial \Omega\right), \quad \text { a.e. } t \in[0, T],
\end{array}
$$

Above, the space $H^{1}\left(\Omega_{\varepsilon}, \partial \Omega\right)$ is the completion, with respect to the norm of $H^{1}\left(\Omega_{\varepsilon}\right)$, of the set of functions $u$ infinitely differentiable in $\bar{\Omega}_{\varepsilon}$, vanishing in a neighborhood of $\partial \Omega$, $\left(H^{1}\left(\Omega_{\varepsilon}, \partial \Omega\right)\right)^{\prime}$ is the dual space of $H^{1}\left(\Omega_{\varepsilon}, \partial \Omega\right)$, and $\langle.,$.$\rangle is the duality product.$

The existence and uniqueness of solutions of (7) is quite classical. It can be obtained using an adaptation the Galerkin method to nonlinear problems: see $[17,18]$ in connection with the method and different nonlinear problems; see, e.g., [12, 13, 14] for different methods in volume perforated media. Note that under the conditions $(3)-(4)(n \leq 4$, respectively) the integral $\int_{S_{\varepsilon}} \sigma\left(x, u_{\varepsilon}\right) v d s_{x}\left(\int_{\Omega_{\varepsilon}} u_{\varepsilon} \vec{v}_{\varepsilon} . \nabla v d x\right.$, respectively) arising in the weak formulation (7) is well defined (cf. [4] and (34)). Also, under these assumptions, we can obtain uniform bounds for the solutions, such as

$$
\left\|u_{\varepsilon}\right\|_{L^{2}\left(0, T ; H^{1}\left(\Omega_{\varepsilon}, \partial \Omega\right)\right)} \leq C,
$$

where $C$ is a constant independent of $\varepsilon$. It should be emphasized that the above estimate is essential both for postulating formal asymptotic expansions of the solution and for obtaining the convergence of the solution (cf. (23) and item C5 in Section 4).

\section{Critical relations and homogenized problems}

In this section, we state the homogenized problems. To obtain these problems, we use asymptotic expansions, matching principles and the technique from the mechanics of continuous media of control volume in thin "coin-like domains" (cf. [10] and references therein). For completeness, in Section 3.1, we present an outline of the proofs. Concerning the justification of the convergence of solutions see Section 4. 
To set the limit problems, let us first introduce the parameters $r_{0}$ and $\beta_{0}$, which can range between 0 and $+\infty$, as

$$
\lim _{\varepsilon \rightarrow 0} \frac{r(\varepsilon)}{\varepsilon^{\frac{n-1}{n-2}}}=r_{0}
$$

and

$$
\lim _{\varepsilon \rightarrow 0} r(\varepsilon) \beta(\varepsilon)=\beta_{0} .
$$

We have the following homogenized problems:

i). The most critical situation occurs when $r_{0}>0$ and $\beta_{0}>0$. In this case, $\beta^{*}$ in (1) is $\beta^{*}=r_{0}^{n-2} \beta_{0}$, and the homogenized problem reads

$$
\begin{cases}\frac{\partial u}{\partial t}-\Delta u+\operatorname{div}\left(u \vec{v}_{0}\right)=f & \text { in }\left(\Omega^{+} \cup \Omega^{-}\right) \times(0, T), \\ u(x, t)=0 & \text { for }(x, t) \in \partial \Omega \times(0, T), \\ {[u(x, t)]=0,\left[\frac{\partial u}{\partial x_{1}}(x, t)\right]=r_{0}^{n-2} \Xi_{\sigma, G_{0}, \beta_{0}}(x, u(x, t))} & \text { for }(x, t) \in \Sigma \times(0, T), \\ u(x, 0)=0 & \text { for } x \in \Omega .\end{cases}
$$

Above, the brackets denote the jump across $\Sigma$ of the enclosed quantities, and the function $\Xi_{\sigma, G_{0}, \beta_{0}}(x, u)$, arising in the transmission condition for the derivatives, is a monotone function of the variable $u$ defined by

$$
\Xi_{\sigma, G_{0}, \beta_{0}}(x, u)=\beta_{0} \int_{\partial G_{0}} \sigma\left(x,\left(W^{x, u}(y)+1\right) u\right) d s_{y},
$$

the extended capacity, where, for each fixed $(x, u), W^{x, u}$ denotes the solution of the "bi-parametric" time-independent local problem

$$
\begin{cases}-\Delta_{y} W^{x, u}=0 & \text { in } \mathbb{R}^{n} \backslash G_{0} \\ u \frac{\partial W^{x, u}}{\partial \nu_{y}}+\beta_{0} \sigma\left(x,\left(W^{x, u}(y)+1\right) u\right)=0 & \text { for } y \in \partial G_{0} \\ W^{x, u}(y) \rightarrow 0 & \text { as }|y| \rightarrow \infty\end{cases}
$$

( $y$ here is an auxiliary variable, cf. also (26)). The function $\Xi_{\sigma, G_{0}, \beta_{0}}(x, u)$ inherits the properties of monotonicity and boundedness of $\sigma(x, u)$ (cf. [10] for the proof as well as for certain technical restrictions; see also item C4 in Section 4). Consequently, once $\vec{v}_{0}$ is defined, the existence of a unique weak solution of (11) holds as that for (6).

The vector function $\vec{v}_{0}$ arising in (11) is the limit, as $\varepsilon \rightarrow 0$, of the velocity $\vec{v}_{\varepsilon}$, cf. $(5)$ and $(23),\left(\vec{v}_{0}, p_{0}\right)$ being the solution of the homogenized Stokes problem:

$$
\begin{cases}-\mu \Delta \vec{v}_{0}+\nabla p_{0}=\vec{F} & \text { in } \Omega^{ \pm} \\ \operatorname{div}\left(\vec{v}_{0}\right)=0 & \text { in } \Omega^{ \pm} \\ \vec{v}_{0}=0 & \text { on } \partial \Omega^{ \pm} \cap \partial \Omega \\ {\left[v_{0 i}\right]=0,} & \text { on } \Sigma, \quad i=1,2, \cdots, n \\ {\left[\mu \frac{\partial v_{0 i}}{\partial x_{1}}-p_{0} \delta_{i 1}\right]=r_{0}^{n-2} \Phi_{i k} v_{0 k}} & \text { on } \Sigma, \quad i=1,2, \cdots, n\end{cases}
$$


where $\delta_{k i}$ is the Kronecker delta, $p_{0}$ is the associated pressure, $\Phi_{i k}, i, k=1,2, \cdots, n$, is the forces matrix, also so-called Stokes capacity matrix, defined from the solutions $\vec{W}_{k}, k=1,2, \cdots, n$ of the Stokes local problems (16) as follows:

$$
\Phi_{i k}=\mu \int_{\mathbb{R}^{n} \backslash G_{0}} \nabla_{y} \vec{W}_{k} \cdot \nabla_{y} \vec{W}_{i} d y
$$

Indeed, $\left(\vec{W}_{k}, Q_{k}\right)$ is the solution of the Stokes problem

$$
\begin{cases}-\mu \Delta_{y} \vec{W}_{k}+\nabla_{y} Q_{k}=0 & \text { in } \mathbb{R}^{n} \backslash G_{0}, \\ \operatorname{div}_{y}\left(\vec{W}_{k}\right)=0 & \text { in } \mathbb{R}^{n} \backslash G_{0}, \\ \vec{W}_{k}=\vec{e}_{k} & \text { on } \partial G_{0}, \\ \vec{W}_{k}(y) \rightarrow 0 & \text { as }|y| \rightarrow \infty\end{cases}
$$

where $Q_{k}$ denotes the associated pressure and $\vec{e}_{k}$ is the unitary vector in the $y_{k^{-}}$ direction $\left(e_{k i}=\delta_{k i}, k, i=1,2, \cdots, n\right)$.

Notice that writing the weak formulation of problem (13), we can show that, cf. Theorem 4.1 in [10], it has a unique weak solution $W^{x, u}$ which belongs to the space completion of $\left\{V \in C^{\infty}\left(\overline{\mathbb{R}^{n} \backslash G_{0}}\right), V\right.$ of compact support $\}$ with respect to the norm $\left\|\nabla_{y} V\right\|_{L^{2}\left(\mathbb{R}^{n} \backslash G_{0}\right)}$, and it satisfies

$$
W^{x, u}(y)=\frac{\mathcal{K}(x, u)}{|y|^{n-2}}+O\left(\frac{1}{|y|^{n-1}}\right) \quad \text { as } \quad|y| \rightarrow \infty,
$$

where $\mathcal{K}(x, u)$ is a constant, independent of $y$, but dependent on the parameters of the problem $x$ and $u$. In addition, we multiply the partial differential equation in (13) by $W^{x, u}$, we apply the Green formula in $B(0, R) \backslash G_{0}$, with $B(0, R)$ any ball containing $\overline{G_{0}}$ and, finally, we take limits as $R \rightarrow \infty$ and we get

$$
-\mathcal{K}(x, u)(n-2) \omega_{n}=-\int_{\partial G_{0}} \frac{\partial W^{x, u}}{\partial \nu_{y}} d s_{y}=\frac{\beta_{0}}{u} \int_{\partial G_{0}} \sigma\left(x,\left(W^{x, u}(y)+1\right) u\right) d s_{y}
$$

where $\omega_{n}$ denotes the area of the unit sphere in $\mathbb{R}^{n}$. Consequently, cf. (12), the strange term in (11) also reads

$$
r_{0}^{n-2} \beta_{0} \int_{\partial G_{0}} \sigma\left(x,\left(W^{x, u}(y)+1\right) u\right) d s_{y}=-r_{0}^{n-2}(n-2) \omega_{n} u \mathcal{K}(x, u) .
$$

ii). In the case where $r_{0}=0$ and $\beta^{*}>0$ in $(1)\left(\beta_{0}=+\infty\right)$, the homogenized problem is

$$
\begin{cases}\frac{\partial u}{\partial t}-\Delta u+\operatorname{div}\left(u \vec{v}_{0}\right)=f & \text { in } \Omega \times(0, T) \\ u(x, t)=0 & \text { for }(x, t) \in \partial \Omega \times(0, T), \\ u(x, 0)=0 & \text { for } x \in \Omega\end{cases}
$$


where $\vec{v}_{0}$ is the solution of the homogenized problem for (5)

$$
\begin{cases}-\mu \Delta \vec{v}_{0}+\nabla p_{0}=\vec{F} & \text { in } \Omega, \\ \operatorname{div}\left(\vec{v}_{0}\right)=0 & \text { in } \Omega, \\ \vec{v}_{0}=0 & \text { on } \partial \Omega .\end{cases}
$$

iii). In the case where $r_{0}=+\infty$ and $\beta^{*}>0$ in $(1)\left(\beta_{0}=0\right)$, the homogenized problem is

$$
\begin{cases}\frac{\partial u}{\partial t}-\Delta u+\operatorname{div}\left(u \vec{v}_{0}\right)=f & \text { in }\left(\Omega^{+} \cup \Omega^{-}\right) \times(0, T), \\ u(x, t)=0 & \text { for }(x, t) \in \partial \Omega \times(0, T), \\ {[u(x, t)]=0, \quad\left[\frac{\partial u}{\partial x_{1}}(x, t)\right]=\beta^{*}\left|\partial G_{0}\right| \sigma(x, u(x, t))} & \text { for }(x, t) \in \Sigma \times(0, T), \\ u(x, 0)=0 & \text { for } x \in \Omega,\end{cases}
$$

where $\vec{v}_{0}=v_{0}^{ \pm}$in $\bar{\Omega}^{ \pm}$is the solution of the Stokes problems in $\Omega^{ \pm}$:

$$
\begin{cases}-\mu \Delta \vec{v}_{0}^{ \pm}+\nabla p_{0}^{ \pm}=\vec{F} & \text { in } \Omega^{ \pm} \\ \operatorname{div}\left(\vec{v}_{0}^{ \pm}\right)=0 & \text { in } \Omega^{ \pm} \\ \vec{v}_{0}^{ \pm}=0 & \text { on } \partial \Omega^{ \pm} .\end{cases}
$$

It should be emphasized that the Stokes problem (5) does not involve the concentration $u_{\varepsilon}$. Consequently, as is well known in the literature (cf. $[1,3,10]$ ), the homogenized results hold for $n \geq 3$ depending on the value of $r_{0}$ in (9). As a matter of fact, in the case where $r_{0}>0$ in $(9)$, we refer to [1] for the convergence

$$
P^{\varepsilon} \vec{v}_{\varepsilon} \stackrel{\varepsilon \rightarrow 0}{\longrightarrow} \vec{v}_{0} \quad \text { in }\left(H_{0}^{1}(\Omega)\right)^{n}-\text { weak, when } n \geq 3
$$

where $P^{\varepsilon} \vec{v}_{\varepsilon}$ is the solution of (5) extended by zero in $G_{\varepsilon}$, and $\vec{v}_{0}$ is the solution of (14). See also $[1,3]$ for $r_{0}=0$ or $r_{0}=\infty$.

\subsection{The asymptotic expansions}

For brevity, in this section, we provide a sketch of the technique and the main ideas to get problems (11)-(13), (19) and (21).

First, on account of (8), we consider an outer expansion for the solution $u^{\varepsilon}$ of (6)

$$
u_{\varepsilon}(x, t)=u^{0}(x, t)+\cdots \quad \text { for } x \in \Omega \backslash \Sigma \times[-d, d], \quad \forall d>0, \quad t \geq 0,
$$

and a local one in a neighborhood of each inclusion $G_{\varepsilon}^{j}$,

$$
u_{\varepsilon}(x, t)=V^{0}(y, t)+\cdots \quad \text { for } y \in B(0, R) \backslash G_{0}, \quad \forall R>0, \quad t \geq 0,
$$

where $y$ is the local variable

$$
y=\frac{x-\widetilde{x}_{\varepsilon}^{\mathrm{j}}}{r(\varepsilon)},
$$


and by dots we denote regular terms in the asymptotic series containing higher-order terms, that we are not using in our analysis. For the velocity field, we use the global approach in $\Omega$, cf. (23),

$$
\vec{v}_{\varepsilon}(x) \approx \vec{v}_{0}(x)
$$

where $\vec{v}_{0}$ is the solution of the homogenized problems (14), (20) and (22) depending on the value of $r_{0}$ in (9).

Second, by matching the local and outer expansion, at the first order, we have

$$
\lim _{|y| \rightarrow \infty} V^{0}(y, t)=\lim _{x \rightarrow \widetilde{x}_{\varepsilon}^{j}} u^{0}(x, t) .
$$

Third, by introducing (24) and (27) in (6), on the basis of $u^{0} \in L^{2}\left(0, T, H_{0}^{1}(\Omega)\right)$, we obtain the following equations for $u^{0}$

$$
\begin{aligned}
& \frac{\partial u^{0}}{\partial t}-\Delta u^{0}+\operatorname{div}\left(u^{0} \vec{v}_{0}\right)=f \text { in }\left(\Omega^{+} \cup \Omega^{-}\right) \times(0, T), \\
& u^{0}=0 \text { on } \partial \Omega \times(0, T), \quad\left[u^{0}\right]=0 \text { on } \Sigma \times(0, T), \\
& u^{0}(x, 0)=0 \text { for } x \in \Omega .
\end{aligned}
$$

Now, to determine completely $u^{0}$, we need to add another transmission condition on $\Sigma$ for the normal derivative of $u^{0}$, which we deduce below depending on the relations between the parameters $\varepsilon, r(\varepsilon)$ and $\beta(\varepsilon)$.

Fourth, we obtain formal asymptotics for $\sigma\left(x, u^{\varepsilon}(x, t)\right)$ in a neighborhood of each inclusion $G_{\varepsilon}^{j}$ :

$$
\sigma\left(x, u^{\varepsilon}(x, t)\right)=\sigma\left(r(\varepsilon) y+\widetilde{x}_{\varepsilon}^{\mathrm{j}}, u^{\varepsilon}(y, t)\right)=\sigma\left(\widetilde{x}_{\varepsilon}^{\mathrm{j}}, V^{0}(y, t)\right)+\cdots .
$$

Then, taking derivatives with respect to $y$ in (6), and using (25), (27) and (30), we obtain that $V^{0}(y, t)$ is a harmonic function in $\mathbb{R}^{n} \backslash G_{0}$, independent of the fluid velocity, and it satisfies

$$
\frac{1}{r(\varepsilon)} \frac{\partial V^{0}}{\partial \nu_{y}}+\beta(\varepsilon) \sigma\left(\widetilde{x}_{\varepsilon}^{\mathrm{j}}, V^{0}(y, t)\right) \approx 0 \quad \text { for } y \in \partial G_{0}
$$

together with (28). Thus, writing $V^{0}(y, t)=u^{0}\left(\widetilde{x}_{\varepsilon}^{\mathrm{j}}, t\right) V(y)$, we look at the dominant terms in the equation on $\partial G_{0}$, and we derive an equation for $V(y)$ on $\partial G_{0}$ that can be Dirichlet, Neumann or a nonlinear Robin condition. In particular, for the most critical situation when $r_{0}>0$ and $\beta_{0}>0, \beta(\varepsilon) r(\varepsilon)=O(1)$ (cf. (10)) and we take

$$
V^{0}(y, t)=u^{0}\left(\widetilde{x}_{\varepsilon}^{\mathrm{j}}, t\right)\left(W^{\widetilde{x}_{\varepsilon}^{\mathrm{j}}, u^{0}\left(\widetilde{x}_{\varepsilon}^{\mathrm{j}}, t\right)}(y)+1\right),
$$

where $W^{\widetilde{x}_{\varepsilon}^{\mathrm{j}}, u^{0}\left(\widetilde{x}_{\varepsilon}^{\mathrm{j}}, t\right)}$ satisfies $(13)$.

Fifth, we use the technique of control volume in thin "coin-like domains" to derive the transmission condition for $u^{0}$. Namely, an integration by parts over the partial differential equation (6) in domains $\left((-\eta(\varepsilon), \eta(\varepsilon)) \times \Sigma_{1}\right) \cap \Omega_{\varepsilon}$, where $\Sigma_{1} \subset \Sigma$ is such that $\partial \Sigma_{1}$ does not touch any inclusion $G_{\varepsilon}^{j}$, and $\eta(\varepsilon)$ satisfies $r(\varepsilon) \ll \eta(\varepsilon) \ll 1$. Using (24), (25), (30) and (32) we get the asymptotic formula

$$
\left.\int_{\Sigma_{1}}\left[\frac{\partial u^{0}}{\partial x_{1}}\right]\right|_{x_{1}=0} d s+\cdots=\beta(\varepsilon) r(\varepsilon)^{n-1} \sum_{\widetilde{x}_{\varepsilon}^{\mathrm{j}} \in \Sigma_{1}} \int_{\partial G_{0}} \sigma\left(\widetilde{x}_{\varepsilon}^{\mathrm{j}},\left(W^{\widetilde{x}_{\varepsilon}^{\mathrm{j}}, u^{0}\left(\widetilde{x}_{\varepsilon}^{\mathrm{j}}, t\right)}(y)+1\right) u^{0}\left(\widetilde{x}_{\varepsilon}^{\mathrm{j}}, t\right)\right) d s_{y} .
$$


Finally, taking limits in (33), as $\varepsilon \rightarrow 0$, provides the transmission condition $u^{0}$ throughout $\Sigma$, which along with (29), gives $u^{0}(x, t)$ the solution of the homogenized problem (11).

To get the homogenized problems (19) and (21), the problem (13) and the formulas (32) and (33) must be suitably modified depending on whether $\beta(\varepsilon) r(\varepsilon) \ll 1$ or $1 \ll \beta(\varepsilon) r(\varepsilon)$.

\section{$4 \quad$ Extensions and concluding remarks}

C1. On the advection term and the dimension $n$. Due to the advection terms $\operatorname{div}\left(u_{\varepsilon} \vec{v}_{\varepsilon}\right)$ in the partial differential equation, cf. (6), and in order to set an integral formulation of the problem in the suitable Hilbert spaces, cf. (7), we need the integral term $\int_{\Omega_{\varepsilon}} u_{\varepsilon} \vec{v}_{\varepsilon} \cdot \nabla v d x$ to be defined $\forall v \in H^{1}\left(\Omega_{\varepsilon}, \partial \Omega\right)$. This happens for the dimension of the space $n=3,4$. Indeed, taking into account that the Sobolev embedding from $H^{1}\left(\Omega_{\varepsilon}, \partial \Omega\right)$ into $L^{q}\left(\Omega_{\varepsilon}\right)$ is continuous when $q \leq \frac{2 n}{n-2}$, we can write

$$
\left|\int_{\Omega_{\varepsilon}} u_{\varepsilon} \vec{v}_{\varepsilon} \cdot \nabla v d x\right| \leq C_{\varepsilon}\left\|\vec{v}_{\varepsilon}\right\|_{L^{n}\left(\Omega_{\varepsilon}\right)}\left\|\nabla u_{\varepsilon}\right\|_{L^{2}\left(\Omega_{\varepsilon}\right)}\|\nabla v\|_{L^{2}\left(\Omega_{\varepsilon}\right)}, \quad \text { when } n \leq 4 \text {. }
$$

The same restriction hold for the weak formulations of the homogenized problems due to the term $\operatorname{div}\left(u \vec{v}_{0}\right)$. Let us refer to [4] for more details about the proof of (34) and to [13] for different restrictions on $\vec{v}_{\varepsilon}$ that could avoid the restriction on $n$ but that might not work when justifying the weak formulation of the homogenized problems, cf. e.g., (14) and (11). Note that the estimate (34) proves to be essential in order to obtain uniform bounds for the solutions, cf. (8), and show convergence. The above restriction on the dimension is not necessary if the advection term does not appear. This happens for instance, when the fluid is at rest (namely, $\vec{v}_{\varepsilon} \equiv 0$ in (6) with $\vec{F}=0$ in (5)), and in models in which a fluid is not involved.

$\mathrm{C} 2$. The stationary problem when $n \geq 3$. In connection with item $\mathrm{C} 1$ above, it should be emphasized that the stationary problem associated with (6) without the advection term have been the subject of research in many papers in the literature when the obstacles or the holes are balls (cf. item C3 below and, e.g., [3, 10, 11] for an extensive bibliography). Hence, for the sake of completeness, we state the homogenized problems when $n \geq 3$ which are also new in the literature.

For the most critical situation when $r_{0}>0$ and $\beta_{0}>0$, we have:

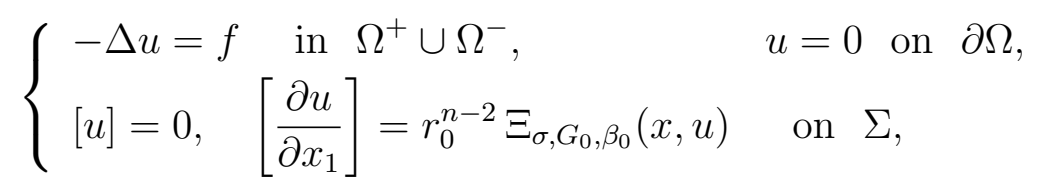

where the strange term $r_{0}^{n-2} \Xi_{\sigma, G_{0}, \beta_{0}}(x, u)$ is defined by (18), cf. also (12).

In the case where $r_{0}=0$ and $\beta^{*}>0$ in (1), we have:

$$
-\Delta u=f \quad \text { in } \Omega, \quad u=0 \text { on } \partial \Omega,
$$


In the case where $r_{0}=+\infty$ and $\beta^{*}>0$ in (1), we have:

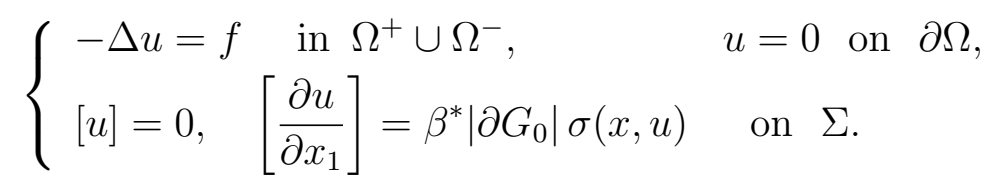

C3. On the geometry of the obstacles. When $G_{0}$ is the unit ball, looking for the solution of $(13)$ as $W^{x, u}(y)=\mathcal{K}(x, u)|y|^{2-n}$, and denoting $H(x, u)=-u \mathcal{K}(x, u)$, the functional equation (18) (cf. also (12) and (17)) can be re-written as

$$
(n-2) H=\beta_{0} \sigma(x, u-H),
$$

while the transmission condition in (11) reads

$$
\left[\frac{\partial u}{\partial x_{1}}(x, t)\right]=r_{0}^{n-2}(n-2) \omega_{n} H(x, u(x, t)) .
$$

Under the assumptions (2)-(3) for $\sigma$, and the additional smoothness $\sigma \in C^{1}(\bar{\Omega} \times \mathbb{R})$, the existence and uniqueness of solution $H(x, u)$ of $(38), H$ smooth and satisfying analogous properties to $\sigma$, has been shown in [4]. Also the proof of the convergence of $u_{\varepsilon}$ towards $u$, as $\varepsilon \rightarrow 0$, in the stationary case, when $G_{0}$ is the unit ball, is in [4] (cf. (35)).

In the case where $\sigma$ is a linear function of $u$, namely, when $\sigma(x, u)=a(x) u$, with $a(x) \geq 0$ a smooth function, the solution $H(x, u)$ of $(38)$ also depends linearly on $u$ : it is defined explicitly by $H(x, u)=\left(a(x) r_{0}+n-2\right)^{-1} a(x) r_{0} u$, where we observe a nonlinear dependence on $a(x)$.

C4. On the nonlinear function $\sigma$. According to $[4,10]$, we note that the hypotheses (2)-(4) on $\sigma$ provide a general framework to obtain an approach for the solution of the scalar problem. Some functions $\sigma(x, u)$ arising in many models (from ecology, hydrogeology, chemical reactions, etc.) for $u \geq 0$, can be suitably extended for $u \leq 0$ in such a way that they satisfy conditions (3) or (4). For instance, this is the case of the adsorption isotherm of Langmuir and Freundlich

$$
\sigma=a u(1+b u)^{-1} \text { and } \sigma=a u^{\gamma}, \quad \text { with } a, b>0,0<\gamma<1
$$

(cf. [4] for further models and references). However, also hypotheses (3)-(4) can be weakened. As it is outlined in [10], the only requirement to be added to the monotonicity of $\sigma$, cf. (2), in order to get the framework of this paper, is that the boundary integrals

$$
\int_{S_{\varepsilon}} \sigma(x, u) v d s_{x} \quad \text { and } \quad \int_{\Sigma} \Xi_{\sigma, G_{0}, \beta_{0}}(x, u) v d s_{x}
$$

define continuous functionals on $H^{1}\left(\Omega_{\varepsilon}\right)$ and $H^{1}(\Omega)$, respectively. 
C5. On the convergence of the solution $u_{\varepsilon}$. In the case where the obstacles are balls, $r_{0}>0, \beta_{0}>0, \sigma \in C^{1}(\bar{\Omega} \times \mathbb{R})(\mathrm{cf} .(38)-(39))$ and $f \in H^{1}\left(0, T ; L^{2}(\Omega)\right)$, the proof of the convergence of (6) is performed by means of an adaptation of the energy method used for the stationary model in [4] (cf. Section II.9 in [18] related to parabolic equations). Indeed, when $G_{0}$ is the unit ball, see also item C3, after introducing a suitable extension operator for $u_{\varepsilon}$ (cf., e.g., [4]), it suffices to take limits in the integral equation associated to (7) using the test functions $v(x, t)=$ $\alpha(t) g(x)-w_{\varepsilon}(x) H(x, \alpha(t) g(x))$, where $\alpha \in C^{1}[0, T], g \in C_{0}^{\infty}(\Omega), H$ is the solution of $(38)$, and $w_{\varepsilon}(x)$ is the function defined as

$w_{\varepsilon}(x)=\frac{|x|^{2-n}-(\varepsilon / 4)^{2-n}}{r(\varepsilon)^{2-n}-(\varepsilon / 4)^{2-n}}$ for $x \in B(0, \varepsilon / 4) \backslash r(\varepsilon) G_{0}, w_{\varepsilon}(x)=1 \quad$ for $x \in r(\varepsilon) G_{0}$,

and it is extended by 0 out of the ball $B(0, \varepsilon / 4)$, and by periodicity to all the balls $B\left(\widetilde{x}_{\varepsilon}^{\mathrm{j}}, \varepsilon / 4\right)$ contained in $\Omega$.

The convergence of the solution $u_{\varepsilon}(x, t)$ of $(6)$, as $\varepsilon \rightarrow 0$, has not been addressed in the literature for the general geometry of the obstacles and the general $\sigma(x, u)$ under consideration (cf. (2)-(4) and item C4 above). When $r_{0}>0$, key points showing convergence in stationary and non-stationary problems will likely be the local problems (cf. (13)). Also, note that proving convergence relies on the construction of suitable extension operators for which additional assumptions on the geometry of the obstacles should be performed. Within the framework of this paper, perforated domains by balls and more restrictive $\sigma$ are assumptions made to obtain the convergence in previous works on stationary and non-stationary models: cf. $[10,11]$ for an extensive bibliography in this connection. The convergence in the general case mentioned above is currently the subject of research. For stationary models without an advection term, the homogenized problems read (35)-(37).

Finally, we refer to [13] for the convergence in a model with an advection term, as in (6), but with the obstacles distributed over the whole volume and with very different assumptions on $\sigma$, on the parameters of the problem, and on the velocity field.

Acknowledgements: The authors would like to thank the anonymous referees for their careful reading of the manupscript and useful comments. The work has been partially supported by MINECO, MTM2013-44883-P.

\section{References}

[1] Allaire, G. Homogenization of the Naviers-Stokes equations in open sets perforated with tiny holes II. Non critical size of the holes for a volume distribution of holes and a surface distribution of holes. Arch. Ration. Mech. Anal. 1983;113:261-298.

[2] Allaire, G, Hutridurga H. Upscaling nonlinear adsorption in periodic porous media: homogenization approach, Appl. Anal. 2016, 95(10): 2126-2161. 
[3] Brillard A. Asymptotic of a viscous and incompressible fluid through a plane sieve. In Bandle C, Bemelmans J, Chipot M, Grüter M, Saint Jean Paulin J, editors. Progress in partial differential equations: calculus of variations, applications (Pont-Mousson, 1991). Vol. 267, Pitman research notes in mathematics series. Harlow: Longman Science and Technology; 1992. p. $158-172$.

[4] Brillard, A. Gómez, D. Lobo, M. Pérez, E. Shaposhnikova, T.A. Boundary homogenization in perforated domains for adsorption problems with an advection term. Appl. Anal. 2016; 95(7):218-237.

[5] Cabarrubias, B. Donato, P. Homogenization of some evolution problems in domains with small holes. Electron. J. Differential Equations 2016; 2016:169.

[6] Cioranescu, D. Donato, P. Ene, H. Homogenization of the Stokes problem with non homogeneous slip boundary conditions. Math. Methods Appl. Sci. 1996;19:857-881.

[7] Cioranescu, D. Murat, F. Un terme étrange venu d'ailleurs I \& II. In: Brezis, H. Lions, J.L. editors. Nonlinear partial differential equations and their applications, Collège de France Séminar, Vols II and III, Pitman Research Notes in Mathematics, Vols 60 and 70, Pitman, London, 1982, p. 98-138, 154-178.

[8] Conca, C. On the application of the homogenization theory to a class of problems arising in fluid mechanics. J. Math. Pures Appl. 1985;64:31-75.

[9] Conca C. Étude d'un fluide traversant une paroi perforée. II. Comportement limite loin de la paroi [Study of fluid flow through a perforated barrier. II. Limit behavior away from the barrier]. J Math Pures Appl. 1987;66:45-70.

[10] Gómez, D. Lobo, M. Pérez, E. Sanchez-Palencia, E. Homogenization in perforated domains: a Stokes grill and an adsorption process, Appl. Anal. 2017; doi: 10.1080/00036811.2017.1395863.

[11] Gómez, D. Pérez, E. Shaposhnikova, T.A. On homogenization of nonlinear Robin type boundary conditions for cavities along manifolds and associated spectral problems, Asympot. Anal. 2012;80:289-322.

[12] Goncharenko, M. The asymptotic behaviour of the third boundary-value problem solutions in domains with fine-grained boundaries. In: Cioranescu, D. Damlamian, A. and Donato, P. editors. Homogenization and applications to material sciences, GAKUTO Internat. Ser. Math. Sci. Appl., Vol. 9, Gakkotosho, Tokyo, 1995, p. 203213.

[13] Goncharenko, M. Khilkova, L. Homogenized model of non-stationary diffusion in porous media with the drift. Zh. Mat. Fiz. Anal. Geom. 2017;13:154-172.

[14] Jagër, W. Neuss-Radu, M. Shaposhnikova, T.A. Homogenization of the diffusion equation with nonlinear flux condition on the interior boundary of a perforated domain: the influence of the scaling on the nonlinearity in the effective sink-source term. J. Math. Sci. 2011;179(3):446-459. 
[15] Kaizu, S. The Poisson equation with semilinear boundary conditions in domains with many tiny holes. J. Fac. Sci. Univ. Tokyo Sect. IA Math. 1989;36:43-86.

[16] Ladyzhenskaya, O.A. The Mathematical Theory of Viscous Incompressible Flow, Mathematics and Its Applications 2, Gordon and Breach, 1969.

[17] Ladyzhenskaya, O.A. Solonnikov, V.A. Ural'tseva, N.N. Linear and Quasi-Linear Equations of Parabolic Type, Providence: American Mathematical Society, 1968.

[18] Lions, J.L. Quelques Méthodes de Résolution des Problémes aux Limites non Linéaires, Dunod, Paris, 1969.

[19] Marchenko, V.A. Khruslov, E.Ya. Homogenization of Partial Differential Equations, Birkhäuser Boston, MA, 2006.

[20] Sanchez-Hubert, J. Sanchez-Palencia, E. Acoustic fluid flow through holes and permeability of perforated walls. J. Math. Anal. Appl. 1982;87:427-453.

[21] Sanchez-Palencia, E. Un problème d'ecoulement lent d'un fluide incompressible au travers d'une paroi finement perforée, In: Bergman, D. Lions, J.L. Papanicolaou, G. et al. editors. Homogenization methods: theory and applications in physics. Collect. Dir. Études Rech. Élec. France, 57, Eyrolles, Paris, 1985, p. 371-400.

[22] Temam, R. Navier-Stokes Equations. Theory and Numerical Analysis. Studies in Mathematics and its Applications, V. 2. North-Holland, Amsterdam, 1979. 